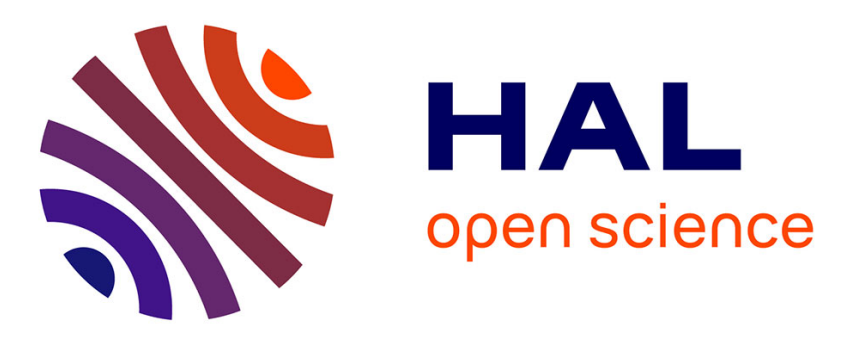

\title{
Supervised learning strategy for classification and regression tasks applied to aeronautical structural health monitoring problems
}

Roberto Miorelli, Andrii Kulakovskyi, Bastien Chapuis, Oscar D'almeida, Olivier Mesnil

\section{To cite this version:}

Roberto Miorelli, Andrii Kulakovskyi, Bastien Chapuis, Oscar D'almeida, Olivier Mesnil. Supervised learning strategy for classification and regression tasks applied to aeronautical structural health monitoring problems. Ultrasonics, 2021, 113, pp.106372. 10.1016/j.ultras.2021.106372 . hal-03163639

\section{HAL Id: hal-03163639 \\ https://hal.science/hal-03163639}

Submitted on 9 Mar 2021

HAL is a multi-disciplinary open access archive for the deposit and dissemination of scientific research documents, whether they are published or not. The documents may come from teaching and research institutions in France or abroad, or from public or private research centers.
L'archive ouverte pluridisciplinaire HAL, est destinée au dépôt et à la diffusion de documents scientifiques de niveau recherche, publiés ou non, émanant des établissements d'enseignement et de recherche français ou étrangers, des laboratoires publics ou privés. 


\title{
Supervised learning strategy for classification and regression tasks applied to aeronautical structural health monitoring problems
}

\author{
Roberto MIORELLI ${ }^{\mathrm{a}, *}$, Andrii KULAKOVSKYI ${ }^{\mathrm{a}}$, Bastien CHAPUIS ${ }^{\mathrm{a}}$, Oscar D'ALMEIDA ${ }^{\mathrm{b}}$, \\ Olivier MESNIL ${ }^{\mathrm{a}}$ \\ ${ }^{a}$ CEA LIST, Centre de Saclay, 91191 Gif-sur-Yvette, France \\ ${ }^{b}$ Safran Tech, 78114 Magny-Les-Hameaux, Francee
}

\begin{abstract}
This paper presents the use of a kernel-based machine learning strategy targeting classification and regression tasks in view of automatic flaw(s) detection, localization and characterization. The studied use-case is a structural health monitoring configuration with an array of piezoelectric sensors integrated on aluminum panels affected by flaws of various positions and dimensions. The measured guided wave signals are post processed with a guided wave imaging algorithm in order to obtain an image representing the health of each specimen. These images are then used as inputs to build classification and regression models. In this paper, an extensive numerical validation campaign is conducted to validate the process. Then the inversion is applied to an experimental campaign, which demonstrate the ability to use a numerically-built model to invert experimental data. DOI: https://doi.org/10.1016/j.ultras.2021.106372

Keywords: Support vector machine, Structural health monitoring, Guided wave imaging, Flaw detection, Flaw sizing, Guided elastic waves
\end{abstract}

\section{Introduction}

Structural Health Monitoring (SHM) relies on the permanent integration of sensors on or inside a structure to monitoring its health. Unlike in Non Destructive Testing (NDT), in which planned maintenance operations must occur periodically, the integration of sensors allows a continuous monitoring of the structure, potentially conducted during its regular exploitation. This leads to substantial benefits of SHM, including a significant cost reduction by shifting from a scheduledmaintenance paradigm to a condition-triggered maintenance, along with the creation of a wealth of information concerning the evolution of the structure overtime and its damage mechanisms, which might then be exploited to lead to more optimized designs and usages. However, the integration of sensors leads to specific difficulties to overcome, among them, ensuring sufficient area coverage with fixed sensors has received significant attention from the literature in the past couple of decades [1].

A widely studied solution to inspect large areas with a limited number of sensors while insuring reasonable defect sensitivity and system reliability, is to use Guided elastic Waves (GW). Indeed, GW propagate over large distances, require low voltage excitation and can be easily measured. Usually in GW-SHM, both actuation and sensing are conducted by lightweight and affordable

\footnotetext{
*Principal Corresponding author

Email address: roberto.miorelli@cea.fr (Roberto MIORELLI)
} 
piezoelectric transducers [2]. The main characteristic of interest of GWs is their highly sensitive propagation behaviour, ensuring that propagated wave-packets will be influenced by defect present on the GW propagation path, but making it potentially difficult to isolate from the other features and characteristics.

To provide both flaw detection and localization of defects, Guided Wave Imaging (GWI) techniques have been developed, leading to images representing the health of the inspected structure. The most common configuration is the following: a plate-like structure is instrumented by a sparse array of $P$ piezoelectric transducers. Each transducer is sequentially harmonically excited to generate GW at a specific frequency, or frequency range. The propagated wave-packets are then measured at every transducer, leading to $P^{2}-P$ measurements. This scan is usually compared to a baseline scan, i.e. an identical scan obtained while the structure is known to be pristine. This baseline comparison is a significant limitation for multiple reasons: first, it means that an already damaged structure can not be retrofitted since the first measurement must be taken in a pristine state and second, the evolution of the signals not induced by defects (e.g. temperature variation, sensor degradation, etc.) must be taken into account to avoid false alarms. Multiple GWI using baseline have been proposed. The simplest of them is RAPID [3] and does not use any knowledge of the inspected material or geometry, allowing for a quick but rough integrity estimate. Taking into account GW propagation speed to triangulate the defect echo to its location, the Delay And Sum (DAS) [4] leads to consistent and reliable imaging results, but neglect the dispersive behavior of the GWs, limiting the resolution. DAS is later upgraded to the Minimum Variance (MV) algorithm [5] by making assumptions on the reflectivity pattern of the inspected defect to better resolve it. The assumption of the defect reflectivity is however quite significant, and the performances of MV are degraded (to the point at which a defect detected by DAS could be missed by MV) if the wrong defect reflectivity pattern is assumed. Finally, using the full knowledge of the dispersion curves, the Excitelet $[6,7,8]$ algorithm provides usually the most resolved and reliable GWI results, but requires the whole propagation Green's function, which depends mainly on the inspected media and the sensor.

To ensure reliable baseline subtraction, Baseline Signal Stretch (BSS) [9] has been proposed to compensate temperature effects by stretching the phase of the signals, but is limited to small temperature differences. It can be used conjointly with Optimal Baseline Subtraction (OBS) [10] to compensate larger temperature differences. OBS requires however to scan the structure multiple times to create a dictionary of baseline signal at various temperatures, which is often unpractical and not necessarily representative of all the potential signal variations. Progress is regularly made on temperature compensation with for example the Dynamic Time Warping (DTW) [11] algorithm, allowing to compensate both phase and amplitude of the signals on a larger temperature interval.

On the other hand, baseline-free GWI techniques exist and consist of attempting to interpret every wave-packet and is therefore limited to fairly simple geometries. For example, GW tomography reconstruction algorithms provide an image quantifying the residual thickness of a potentially corroded area but require a large number of sensors $[12,13]$. Non quantitative imaging can also be achieved with Compressed Sensing based GWI [14, 15]. Overall, baseline-free GWI techniques are very promising but require more sensors than baseline-reliant techniques.

In this work, the baseline GWI technique DAS, being the most common baseline-GWI technique in the literature, is used. However this algorithm (and baseline-reliant GWI in general) provides only the detection and the localization of flaws while no information is directly provided about the size of the defect, which is however essential to keep track of its evolution and raise a red flag when a detected defect reaches a critical size. It has been demonstrated that the DAS images are sensitive to defect size [16] but no analytical link can be identified between the damage index 
values of a DAS image and the defect size. Furthermore, the detection and localization steps are always done manually and visually, meaning that a trained operator must analyze the GWI results to provide a diagnostic.

In the NDT community, there have been various efforts aiming at applying Machine Learning (ML) classification and regression tasks based on ultrasounds testing signals [17, 18] and eddy current testing signals [19, 20]. Nevertheless, the application of ML strategy to post-processed GW-SHM signals targeting flaw(s) localization and sizing has been not deeply studied yet.

This paper proposes a model-driven supervised ML inversion strategy trained on numerical simulations degraded by an ad-hoc noise model to account for the sensor degradation, variation of temperature and measurement noise. Multiple ML classifiers are assessed to compare detection performance. Quasi-real time inversion of both defect localization and sizing is performed on experimental data, thus showing the capacity of the method to invert experimental data while being exclusively trained on numerical data.

The developed method is applied to an aluminium panel instrumented by 8 piezoelectric transducers and damaged by a through-hole. The authors acknowledge the simplicity of the use-case under consideration, which serves the sole purpose of illustrating the application of the method without constraining its genericity. At the likely cost of a larger training database and a different hyper-parameters choice, the method is largely applicable to more complex structures. Actual performance of the method would then vary depending on the capacity of the method to extract the relevant features from the data for each specific use-case.

The outline of the paper is as follows. Firstly, the forward model used to generate the data is presented along with a synthetic model of noise aiming at introducing degradation in the raw simulated GW signals which mimics the behaviour of noisy measurements and degrading piezoelectric (PZT) sensors. Then, a quick description of the DAS GWI technique is provided and discussion on the sensitivity of the GW signals with respect to the simulation parameters under consideration (e.g., flaw size and position) is provided. Subsequently, is introduced the supervised learning strategy adopted in this paper by providing a detailed description of the dimensionality reduction and the model fitting procedures adopted. In the last part, an exhaustive numerical validation campaign is provided along with experimental measurements carried out in a laboratory is provided and discussed. Conclusions and further research perspectives close the paper.

\section{Guided Wave Imaging}

Guided Wave Imaging (GWI) is a fast, reliable and efficient method for the damage detection and localization in plate-like structures. It can be implemented using a sparse embedded array of inexpensive and low energy consumption piezoelectric transducers. As such, this method is a natural choice for GWs-based SHM system, but the knowledge of the pristine state of the structure, e.g. baseline, is often required.

Baseline approaches are based on the comparison between reference signals and signals at the current time, in which the state of the structure is unknown. Such comparison can be performed in several ways, including GWs signals subtraction where residual signals are computed as the difference between current and baseline signals. Residual signals are then processed by GWI algorithms in order to compute an image representing the integrity of the structure. In this work, piezoelectric transducers are placed in a circle surrounding the region of interest to be interrogate defects from multiple directions and, therefore, to maximize its detectability.

This section starts with an explanation of the numerical model, herein called the forward model and integrated to the CIVA software, which is used to generate the GWs signals database. It is 
followed by the GWI algorithm Delay-And-Sum [4] that is used for processing GWs signals in order to compute a dataset of images representing the integrity of the structure. Finally, a parametric noise model for GWs signals deterioration is presented.

\subsection{Forward model}

Regardless of the problem, machine learning methods generally require a significant amount of data to build an accurate model. Obviously, an experimental database of GWI samples would be advantageous in comparison with a simulated one as it would reproduce operational disturbances, but its generation cost is prohibitive due to the large number of samples required. Therefore, it is chosen to generate a numeric database of GWs signals that are then deteriorated using a synthetic noise model in order to assimilate numeric GWs signals with their experimental counterparts.

The Finite Element (FE) GWs simulations methods are usually time consuming due to the small element size and time step requirements [21]. In this work, the forward model is based on the transient Spectral Finite Element (SFE) method implemented in the CIVA software as this method allows to overcome the aforementioned limitations while being computationally efficient [22, 23]. For the sake of brevity, only the main steps of the SFE method are presented here but more details can be found in [24]. The SFE method relies on two main concepts to decrease the computational time and the memory footprint. First, a high order spectral finite element method is implemented to significantly reduce the number of elements [25]. Secondly, a macro-element pre-meshing strategy is used to optimize the mesh considering its identical regions and an implicit element orientation. This leads to a significant reduction of CPU load and memory footprint, which allows running multiple simulations either in parallel or sequentially and enables the generation of an extensive database of GWs signals in a reasonable amount of time.

The studied configuration is a square aluminum plate of $600 \times 600 \times 3 \mathrm{~mm}^{3}$ instrumented by eight circular piezoelectric transducers $18 \mathrm{~mm}$ in diameter that are evenly located on a circle of $300 \mathrm{~mm}$ in diameter. The excitation frequency is $40 \mathrm{kHz}$ and it is assumed that the transducer applies an axisymmetric radial load at the surface of the panel according to Crawley's model [26]. The studied defect is a single circular through-hole of various positions and sizes for each sample. For this study the position of the defect is limited to being inside the circle of transducers. The simulation is conducted for every transducer excitation sequentially and on a duration of $300 \mu$ s. The total simulation time of the eight sequential transducers excitations is about five minutes on a standard laptop, equipped with Intel Xeon E3 - 1505M processor with 32 GB of RAM, and requires a memory footprint of about $100 \mathrm{MB}$.

\subsection{Parametric synthetic noise model}

The forward model accurately simulates GWs propagation and GWs-defect interaction but does not reproduce operational disturbances. Hence, GWI samples computed using numeric database might not completely be representative of experimental GWI process. In order to overcome this limitation, a parametric noise model is developed and applied in order to deteriorate the database of simulated GWs signals in coherence with the operational conditions. Taking into consideration that GWI method used in this work relies on residual signals, the synthetic noise model is applied to GWs signals representing both pristine and damaged states.

This model assumes that a GW signal $s^{k}(t)$ can be distorted by several factors, including the temperature variation, transducers bounding degradation and acquisition noise. It is expressed as follows:

$$
s_{\text {deg }}^{k}(t)=\gamma s_{\text {orig }}^{k}(t(1+\chi))+\nu
$$


where $\nu$ represents the acquisition noise, $\chi$ is a stretching factor of time axis $t, \gamma$ is amplitude variation parameter, $s_{\text {orig }}^{k}(t)$ is the original and $s_{d e g}^{k}(t)$ is the deteriorated GWs signals for the $k$-th transducers pair.

The temperature difference between pristine and current states modifies the group and phase velocities of propagating guided modes. In a first order approximation, this effect consists in linear time vector stretching, which is controlled by the $\chi$ parameter. The amplitude of GWs signals may vary over time due to transducers bounding degradation and is modelled by the parameter $\gamma$. Finally, acquisition noise is considered as the white noise and is controlled by the parameter $\nu$. On the basis of experimental observations, all the three deterioration parameters are supposed to vary in the following ranges complying with random uniform distributions: $\gamma \subset[0.875,1.125]$, $\chi \subset\left[-5 \mathrm{e}^{-4}, 5 \mathrm{e}^{4}\right]$ and $\nu \subset\left[0,2 \mathrm{e}^{-2}\right]$. These parameter ranges were selected to approximately cover a degradation range of GWs signals that were observed experimentally while performing defect imaging under varying temperature with a maximal temperature difference of about $20{ }^{\circ} \mathrm{C}[27]$.

Note that the degradation model does not modify the modal content of the wavepackets, which is dependent in the forward model of the dimension of the transducer and the propagating wavelength $[26,28]$. In addition, for the configuration under study, both A0 and S0 modes coexist at comparable amplitudes in the signals. Even though the imaging process described in the following section assumes the presence of a single A0 mode, the S0 does not significantly degrade the results as it interfere destructively with itself in the imaging process. Hence, it is expected that a minor mode amplitude variation would not alter the results significantly.

\subsection{Delay and sum imaging}

Consider a set of $P$ piezoelectric transducers distributed over the structure. Following the round-robin process, each transducer is driven with harmonic excitation for GWs generation in the structure while all the others are used for sensing. In total $P \times(P-1)$ signals are collected, and residual signals are obtained for each $k$ pair of transducers by subtracting the current state $s_{c}^{k}(t)$ from the baseline $s_{b}^{k}(t)$. It is expressed as follows:

$$
s_{r}^{k}(t)=s_{c}^{k}(t)-s_{b}^{k}(t)
$$

For every material point $(x, y)$ of the inspected area, a Damage Index (DI) value is computed as follows. First, the envelopes of the analytical residual signals are calculated via the Hilbert transform:

$$
r^{k}(t)=\sqrt{s_{r}^{k}(t)^{2}+\hat{H}\left(s_{r}^{k}(t)\right)^{2}}
$$

where $r^{k}(t)$ is the envelope of the residual signal and the operator $\hat{H}$ denotes then Hilbert transform. Then, the time of flight of the GWs is computed as follows:

$$
t^{k}(x, y)=\frac{\sqrt{\left(x-x_{i}\right)^{2}+\left(y-y_{i}\right)^{2}}}{C_{g}}+\frac{\sqrt{\left(x-x_{j}\right)^{2}+\left(y-y_{j}\right)^{2}}}{C_{g}}
$$

where $t^{k}(x, y)$ is the time of flight of the GW that propagates with a group velocity $C_{g}$ from the transmitting transducer $i$ located at $\left(x_{i}, y_{i}\right)$ to the inspected point $(x, y)$ and from the latter to the receiving transducer $j$ at $\left(x_{j}, y_{j}\right)$. The guided mode's group velocity $C_{g}$ can be determined using various simulation frameworks, such as the semi-analytical finite elements method [29], or it can be experimentally measured. Finally, each residual signal is delayed by the corresponding time of 


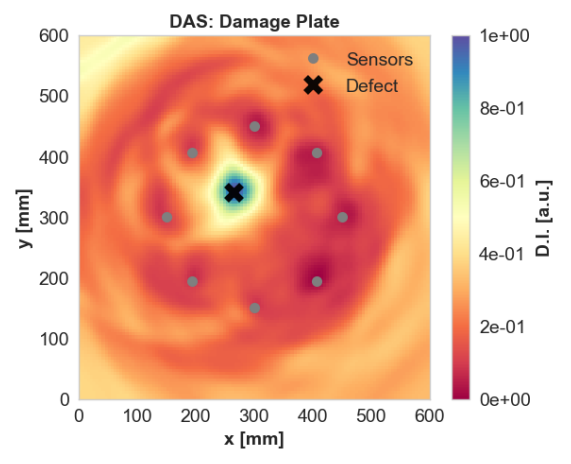

a)

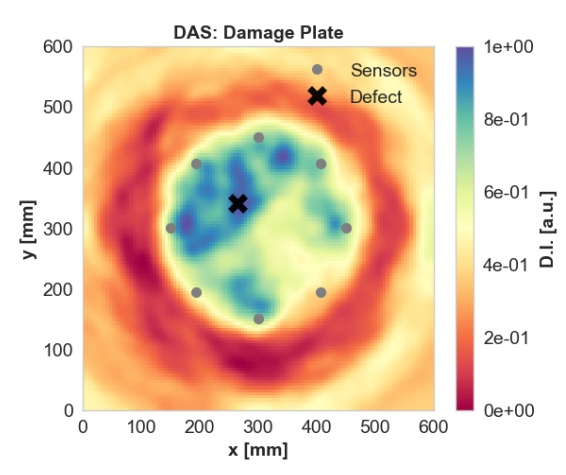

b)

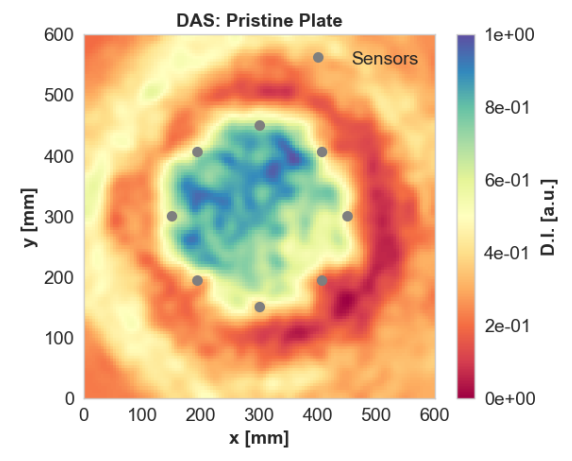

c)

Figure 1: Examples of GWI results in a aluminium plate at $40 \mathrm{kHz}$ using GWs signals simulated by the SFE method. GWIs computed results for a $4.72 \mathrm{~mm}$ radius through-hole are given in $a$ ) based on the raw numerical signal, in $b$ ) accounting the parametric synthetic noise model and in $c$ ) results of a pristine aluminium panel when the parametric synthetic noise model is employed.

flight, so that each point of the image can be associated with a value of the envelope. For each material point, the DI value is computed as follows:

$$
D I^{D A S}(x, y)=\sum_{k=1}^{P(P-1)} r^{k}\left(t^{k}(x, y)\right) .
$$

Since guided waves are dispersive, this algorithm is better suited to narrow-band signals to limit the dispersion effects. In practice, a compromise must be found between narrow-band and temporal resolution.

For illustration purposes, GWI results computed with simulated GWs signals in the studied configuration without application of the degradation model is show in Figure 1a. In this image, the defect imaging is performed at $40 \mathrm{kHz}$ by the DAS algorithm using the $A_{0}$ mode and the structural flaw is a through-hole $4.72 \mathrm{~mm}$ in radius. The GWI results using the original simulated GWs signals, shown in Fig. 1a, is compared to the same use-case where the synthetic noise model has been applied to deteriorated GWS signals in Figure 1b. It can be observed that the imaging quality has significantly decreased and the defect is partly concealed by imaging artifacts. Figure 1c presents the GWI result where the synthetic noise model is applied on GWs signals that represent a pristine structure. In other words, it represents what could be obtained experimentally while imaging pristine plate and might lead to false alarms.

\subsection{Inverse problem analysis}

While a single DI value does not provide sufficient information about the integrity of the structure, the spatial distribution of the DI values can be interpreted for determining the presence of the defect, its location and size. Damage detection and localization are straightforward from the visual analysis of the intensity distribution in GWI results based on noise-free signals. On the other hand, with noisy signals, flaw(s) detection and localization can be trickier to perform. Moreover, damage sizing is a more complex problem that cannot be solved visually.

Consider the same GWI configuration as presented in Section 2.1. The SFE method is used to simulate GWs propagation and GWs-defect interaction in the plate whereas the structural damage is represented by a through-hole located at $(x=250 \mathrm{~mm}$ and $y=280 \mathrm{~mm})$, that progressively grows from $2.5 \mathrm{~mm}$ to $8.0 \mathrm{~mm}$ in radius with increments of $0.5 \mathrm{~mm}$. The inspection is conducted at $40 \mathrm{kHz}$ corresponding to a interrogating wavelength of $\lambda_{A_{0}}^{40 \mathrm{kHz}}=24.8 \mathrm{~mm}$. The corresponding GWI 


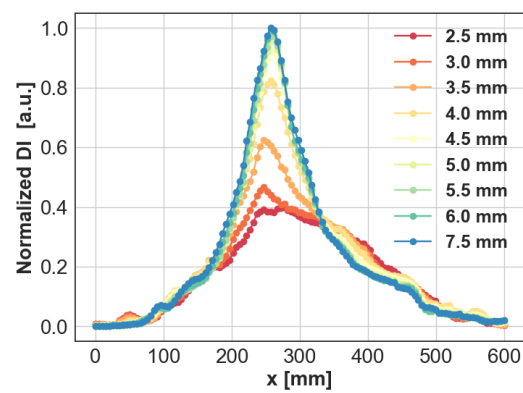

a)

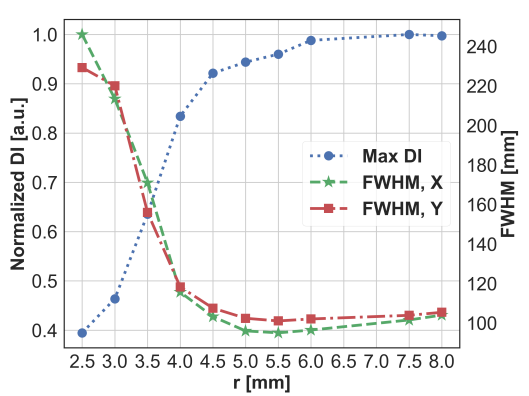

b)

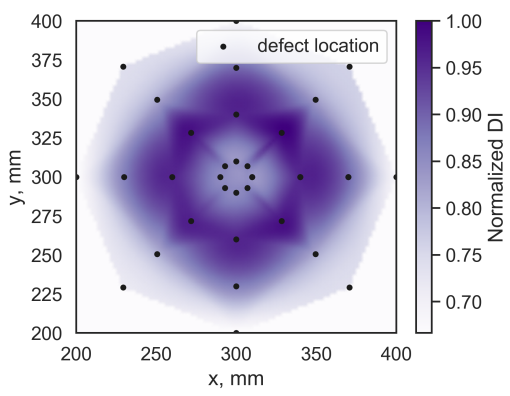

c)

Figure 2: Demonstration of GWI sensitivity to the damage size at $40 \mathrm{kHz}$. In $a$ ) normalized defect DI values profiles and in $b$ ) normalized maximum of DI (Max DI) and Full Width Half Maximum (FWHM) versus hole radius. In c), Third order polynomial interpolation of the normalized maximum of DI values distribution over the plate for defects $5 \mathrm{~mm}$ in radius at various locations.

results are normalized with respect to the highest DI value, and the DI profiles through the damaged region are presented in Figure 2a. It can be observed that for small defects $(R \subset[2.5,5.5] \mathrm{mm}) \mathrm{DI}$ values increase] progressively and sharpen with the radius of the through-hole but for larger ones reach a plateau and even decrease in magnitude a little.

It is worth noting that there are regions where the defect size versus maximal DI relation is almost linear $(D I \subset[2.5,4.5] \mathrm{mm}$ and $D I \subset[6.0,8.0] \mathrm{mm})$ but overall, these variations are non-linear in both height and width.

It should also be pointed out that DI values distribution is not spatially homogeneous, meaning that DI values differ for the same size defects located at various positions. To demonstrate this effect, thirty two SFE simulations representing transverse holes $5 \mathrm{~mm}$ in radius at different locations are run and post-processes by means of the DAS algorithm. The maxima of DI values are then collected for the corresponding locations and interpolated using $3^{\text {rd }}$ order polynomial interpolation in order to construct a map representing the maximum DI for each defect position, as represented in Figure 2c). Furthermore, it can also be observed that DI values' distribution is conditioned by the geometry of the structure and transducers' arrangement.

Taking into account these two non-linear geometrical behaviors of DI values distribution regarding the defect location and size variations, the defect sizing problem cannot be inverted directly from GWI results.

In the next section a data-driven approach, i.e. a supervised machine learning strategy, is proposed in order to handle such a complex non-linear relationship in view of performing classification and regression tasks.

\section{Supervised learning schema}

Numerical software can be exploited to simulate a collection of input-target couples, i.e. labelled data, that can be employed in a learning procedure avoiding as much as possible expensive measurement campaigns. A ML paradigm based on the use of inputs-targets relationships is known under the name of supervised learning. The set of input-target couples employed to fit the model is known as training set. In this paper, the developed ML strategy considers as inputs a set of post-processed guided wave signals and as targets the respective classes or parameters (e.g., flaw types, flaw(s) size/position, specimen, geometry, etc.). In the following, we refer to it as ML-based classification or inversion/regression procedure, respectively. That is, depending on the target 
type, classification or regression tasks can be distinguished. In the framework of NDT, classification algorithms provides discrete-valued predictions such as damage detection and flaw(s) type discrimination, for instance. On the other hand, when regression tasks in view of inversion are performed, the outcome of the ML algorithm is a continuous set of values that can be exploited in order to perform flaw(s) localization or characterization.

The training set is composed of $N$ samples having as input a matrix of $N \times M$ items where $M$ stands for the size of the guided wave images (i.e., the inputs) whereas the outputs or targets (i.e., the classes or parameters) are given by a set of $N \times O$ values. For classification tasks, $O$ is a scalar value, while for regression, $O$ is a vector which dimensions correspond to the space of parameters to be retrieved in the inversion procedure. More into details, the input space is denoted through the vector $\mathbf{x}$ such that $\mathbf{x} \subseteq \mathbb{R}^{M}$ while the target space is described through the vector $\mathbf{y}$ with $\mathbf{y}=\{-1,1\}$ or $\mathbf{y} \subseteq \mathbb{R}^{O}$ in the case binary classification or regression, respectively. The training set is defined as the collection of inputs-targets pairs as

$$
S_{t r n}=\left\{\left(\mathbf{x}_{1}, \mathbf{y}_{1}\right), \ldots,\left(\mathbf{x}_{N}, \mathbf{y}_{N}\right)\right\} \subseteq\left(\mathbf{X}_{t r n} \times \mathbf{Y}_{t r n}\right)^{N}
$$

where the $i$-th couple $\left(\mathbf{x}_{i}, \mathbf{y}_{i}\right)$ represents the $i$-th inputs vector (also known as example or instance) and the associated $i$-th target (also known as label) within $S_{t r n}$, respectively [30].

The envisaged learning procedure should be carefully considered to get the best-possible performance. For example, in the studied configuration, the number of $M$ pixels for each image is very large and it is, for regression cases, associated to a vector of $O$ components. Therefore, the ML strategy should be able generalize on unseen examples i.e., avoid under- or over- fitting and it should be as efficient as possible in both the training and the testing phases. In the next sections, the strategy developed in order to obtain an efficient and robust classification and regression ML procedure applied on GWI data is introduced.

\section{Dimensionality reduction stage via principal components analysis}

Due to the nature of the input of the training phase, the size of the training set $N \times M$ is large, which can lead to a slow training and an accurate solution (i.e., model) might be hard to find. This problem is known in the ML community under the name of curse of dimensionality. In order to tackle this issue, the dimension of the data is reduced by projecting them onto the space of the extracted or projected features which spans a smaller cardinality. This procedure is commonly referred as dimensionality reduction or data decomposition. In this paper, the Principal Component Analysis (PCA) is employed in order to project the original data associated to the imaging results into a subspace of $J \ll M$ principal components also called latent or extracted components. In such a subspace, the variance of the data is maximized [31, 30].

Let us consider the inputs of the training set (6) associated to the set of $N$ images of $M$ pixels.

The matrix $\mathbf{X}_{t r n} \in \mathbb{R}^{N \times M}$ represents the whole set of images after vectorization. For sake of simplicity it is assumed that $\mathbf{X}_{t r n}$ is a zero-mean matrix. In order to obtain the PCA projection of the training set input samples the covariance matrix of the training is built as in [31].

$$
\mathbf{C}=\frac{\mathbf{X}_{t r n}^{\top} \mathbf{X}_{t r n}}{N}
$$

The eigen-decomposition of (7) is then performed such that:

$$
[\mathbf{U}, \Lambda]=\operatorname{eig}(N \mathbf{C}),
$$


where the pair of matrices on the right hand side of the equation are the results of the eigenvalue decomposition such that the columns of matrix $\mathbf{U}$ represent the eigenvectors and $\Lambda$ is the diagonal matrix that has for elements the eigenvalues of $C$ such that $C \mathbf{U}=\mathbf{U} \Lambda$. Therefore, in (8), each element of the training set is projected onto the subspace spanned by the first $J$ eigenvectors as

$$
\tilde{\mathbf{x}}_{i}=\mathbf{U}_{J}^{\top} \mathbf{x}_{i}
$$

with $i=1, \ldots, N$, thus the training set projected onto the feature space is given as $\tilde{\mathbf{X}}_{t r n}=$ $\left\{\tilde{\mathbf{x}}_{1}, \ldots, \tilde{\mathbf{x}}_{N}\right\} \in \mathbb{R}^{N \times J}$. Since the eigenvalues contained into the columns of $\mathbf{U}$ are ordered in a decreasing manner, the space spanned by the $J$-th first extracted principal components is the one that maximize the variance of the data contained into $\mathbf{X}_{t r n}$. The choice of $J$ is then critical in order to be able to capture the largest possible variance on the data providing the smallest possible $J$ to limit dimensionality. A way to handle this trade-off consists in considering the so-called explained variance which is defined as the ratio between the first $M$ eigenvalues and the sum of all the eigenvalues

$$
\text { explained variance }=\frac{\sum_{j=1}^{J} \lambda_{j}}{\sum_{j=1}^{M} \lambda_{j}},
$$

where $\lambda_{j}$ is the $j$-th eigenvalue within $\Lambda$.

The number of features is retrieved by fixing the desired explained variance value, usually set between 0.95 and 0.99 .

\section{Learning and prediction phases based on support vector machine algorithm for classification and regression tasks}

In this section, the ML algorithm employed for classification and regression tasks based on the SHM images is introduced. In Section 3, it is showed that the relationships between inputs and DIs are non-linear as a function of both the defect size and the defect position. Since the PCA feature extraction consists in a linear transformation of the data, the relationships between extracted features and defect parameter remains non-linear. Therefore, the extracted features from PCA are mapped into an alternative feature space, i.e., the space of kernels, where the inputstargets relation behaves linearly. In the following subsections, a mathematical overview on how the images, with or without dimensionality reduction, are mapped into the kernel space is provided. In the literature, the kernel space is also referred as feature space, nevertheless this nomenclature is avoided here in order to avoid misconception as we already referred to it for the "PCA" extracted features. Subsequently, the kernel based extension of Support Vector Machine (SVM) algorithm for classification and regression tasks is introduced from a theoretical point of view.

\subsection{Mapping the extracted features into the kernel space}

Mapping data into a high-dimensional feature space enables to perform linear pattern analysis and thus exploit any linear classification or regression algorithm for nonlinear tasks [32]. Referring to the PCA space associated to the training set introduced in the previous section, a mapping function $\phi$ that projects the training set $\tilde{\mathbf{X}}_{t r n}$ to an inner product feature space $\mathscr{F}$ is defined as in $[32]$ :

$$
\phi: \tilde{\mathbf{x}} \in \mathbb{R}^{J} \longmapsto \phi(\tilde{\mathbf{x}}) \in \mathscr{F} \subseteq \mathbb{R}^{F},
$$

the choice of $\phi$ is such that non-linear relations in $\tilde{\mathbf{x}}$ becomes linear in $\phi(\tilde{\mathbf{x}})$. Therefore the training set projection into the feature space is obtained by applying on each inputs the transformation (11). Then Equation (6) turns into 


$$
\hat{S}_{t r n}=\left\{\left(\phi\left(\tilde{\mathbf{x}}_{1}\right), \mathbf{y}_{1}\right), \ldots,\left(\phi\left(\tilde{\mathbf{x}}_{N}\right), \mathbf{y}_{N}\right)\right\} \subseteq\left(\tilde{\mathbf{X}}_{t r n} \times \mathbf{Y}_{t r n}\right)^{N}
$$

Assuming a linear relationship in the kernel space, the most suitable pattern function fitting this requirements is the one that minimize the discrepancy $\xi$ between the regression results and the ground truth i.e., $|\xi|=\left|\mathbf{y}_{i}-g\left(\phi\left(\tilde{\mathbf{x}}_{i}\right)\right)\right|=\left|\mathbf{y}_{i}-\left\langle\mathbf{w}_{i}, \phi\left(\tilde{\mathbf{x}}_{i}\right)\right\rangle\right|$, where $i=1, \ldots, N$ and $\mathbf{w}_{i}$ is the $i$-th weight vector. One can show [30] that the calculation of $\mathbf{w}$ can be performed such that $\mathbf{w}=\tilde{\mathbf{X}}_{t r n}^{\top} \alpha$ where $\alpha=\left(\tilde{\mathbf{X}}_{t r n}^{\top} \tilde{\mathbf{X}}_{t r n}\right)^{-1}=\mathbf{G}^{-1}$. $\mathbf{G}$ is referred as the Gram matrix which is a $N \times N$ symmetric semi-definite positive matrix. The entries of $\mathbf{G}$ are given by the kernel function [32]

$$
k\left(\tilde{\mathbf{x}}_{i}, \tilde{\mathbf{x}}_{j}\right)=\mathbf{G}_{i j}=\left\langle\phi\left(\tilde{\mathbf{x}}_{i}\right), \phi\left(\tilde{\mathbf{x}}_{j}\right)\right\rangle,
$$

where $\langle\cdot, \cdot\rangle$ denotes the inner product. The mapping onto the feature space that can possibly have an infinite number of dimensions, can be performed without calculating the computationally expensive function $\phi(\cdot)$. Such a computational escamotage is also known as kernel trick [32]. In this work, the Gaussian kernel function is employed and defined as

$$
k\left(\tilde{\mathbf{x}}_{i}, \tilde{\mathbf{x}}_{j}\right)=\exp \left(\frac{-\left\|\tilde{\mathbf{x}}_{i}, \tilde{\mathbf{x}}_{j}\right\|^{2}}{2 \sigma^{2}}\right)
$$

where $\sigma$ is the kernel hyper-parameter to be tuned. It worth mentioning that, even though the Gaussian kernel is widely used in the ML community. Other kinds of kernels can be employed or design to possibly better address the problem at hand [32].

\subsection{Nonlinear support vector machine for classification and regression tasks}

Thanks to the kernel trick, a non-linear relationship can be fitted between the inputs and targets passing through the representation of the data in the feature space. This space is given by the inner product between the projected samples in the space spanned by the PCA components. Different kernel-based classification or regression models can be fit to the data but in this paper, the SVM algorithm is employed since it guarantees good generalization performance on noisy experimental data [30]. Moreover, due to its mathematical background rooted in the statistical learning theory, SVM provides a deeper insight on the choice of hyper parameters than other kernel based method such as kernel ridge regression or neural networks. Finally, SVM also enables sparse classification and regression models that lead to a more lightweight and efficient predictions stage.

In classification problems, although the corresponding decision boundary is not linear, it may happen that data points are separable by hyper-planes e.g., classes are slightly overlapped. SVM for classification (referred in the following also as SVC) allows to neglect miss-classified data that are on the "wrong" side of the boundary by introducing a slack variable $\xi_{n} \geq 0$ with $n=1, \ldots, N$. When $0 \leq \xi_{n} \leq 1$, the data laying on the class boundaries or inside the correct class, whereas the $\xi_{n}>1$ represents miss-classified samples. One can show [30] that such a kind of SVM classifier, known as soft-margin SVM, is fit onto the feature space data by maximizing the Lagrangian in its dual form $\mathcal{L}(\alpha)=\sum_{i=1}^{N} \alpha_{i}-\frac{1}{2} \sum_{i, j=1}^{N} \alpha_{i} \alpha_{j} y_{i} y_{j} k\left(\tilde{\mathbf{x}}_{i}, \tilde{\mathbf{x}}_{j}\right)$ subject to $\sum_{i=1}^{N} \alpha_{i} y_{i}=0$ and $C \geq \alpha_{i} \geq 0$ with $i=1, \ldots, N[33]$ where $C$ behaves as a penalty parameter. The dual variable non-zero coefficients $\alpha_{s}$ are calculated via quadratic programming minimization. Subsequently, the decision on an unseen input vector $\tilde{\mathbf{x}}_{t}$ belonging to the $t$-th row of the test set made of $T$ samples $\tilde{\mathbf{X}}_{t s t} \in \mathbb{R}^{T \times J}$ is obtained by 


$$
\hat{y}_{t}=\sum_{s=1}^{N_{S V}} \alpha_{s} y_{s} k\left(\tilde{\mathbf{x}}_{t}, \tilde{\mathbf{x}}_{s}\right)+b,
$$

where $N_{S V}$ represents the number of Support Vectors (SVs). The SVs represent the subset of inputs-targets training set samples employed for prediction purposes. The SVs are associated to the non-zero $\alpha_{i}$ values. The bias parameter $b$ in (15) is estimated as $b=\frac{1}{N_{S V}} \sum_{s=1}^{N_{S V}}\left(y_{s}-\tilde{\mathbf{x}}_{s} \mathbf{w}\right)$ where $\mathbf{w}$ is given as $\mathbf{w}=\sum_{i=1}^{N} \alpha_{i} y_{i} \tilde{\mathbf{x}}_{i}$ [33].

For regression tasks, SVM provide an elegant way to obtain sparse solutions by employing the so-called $\varepsilon$-insensitive error function. Particularly, starting from the primal formulation of the Lagrangian and by exploiting the Karush-Kuhn-Tucker condition on the primal variable one can show that the dual variable Lagrangian to be maximized for regression is given by [33]

$$
\begin{aligned}
\mathcal{L}\left(\alpha, \alpha^{*}\right)= & \frac{1}{2} \sum_{i, j=1}^{N}\left(\alpha_{i}-\alpha_{i}^{*}\right)\left(\alpha_{j}-\alpha_{j}^{*}\right) k\left(\tilde{\mathbf{x}}_{i}, \tilde{\mathbf{x}}_{j}\right) \\
& -\sum_{i=1}^{N}\left(\varepsilon-y_{i}\right) \alpha_{i}-\sum_{i=1}^{N}\left(\varepsilon+y_{i}\right) \alpha_{i}^{*},
\end{aligned}
$$

subject to the constraint $\sum_{i=1}^{N}\left(\alpha_{i}-\alpha_{i}^{*}\right)=0$ with $0 \leq \alpha_{i}^{(*)} \leq C$. The learning task consists in retrieving the $\left(\alpha_{s}, \alpha_{s}^{*}\right)$ with $s=1, \ldots, N_{S V}$ non-zero pairs which represents the SVs. By employing quadratic programming minimization algorithms, the SVs are retrieved and one can perform regressions on an unseen input vector $\tilde{\mathbf{x}}_{t}$ belonging to the $t$-th row of the test set $\tilde{\mathbf{X}}_{t s t} \in \mathbb{R}^{T \times J}$ as follows

$$
\hat{y}_{t}=\sum_{s=1}^{N_{S V}}\left(\alpha_{i}-\alpha_{i}^{*}\right) k\left(\tilde{\mathbf{x}}_{t}, \tilde{\mathbf{x}}_{s}\right)+b,
$$

where, in practice, $b$ can be retrieved by averaging over all estimates as $b=\frac{1}{N_{S V}} \sum_{i=1}^{N_{S V}} y_{i}-\varepsilon-$ $\sum_{j=1}^{N_{S V}} k\left(\tilde{\mathbf{x}}_{i}, \tilde{\mathbf{x}}_{j}\right)$ [33]. In the Subsection 6.1, more details will be provided about the tuning of SVM hyper-parameters (e.g., $C$ and $\varepsilon$ ) needed to achieve a high accuracy classification or regression model.

\section{Numerical and experimental validation}

In this section, results obtained by applying the ML framework introduced in the previous sections are presented and analyzed. Firstly, a classification procedure targeting detection tasks based on numerical data degraded by the model introduced in Section 2.1 is presented. Subsequently, the model fitting procedure targeting the estimation of flaw position and sizing is provided.

\subsection{Problem description, experimental set-up, calibration procedure and training phase procedure}

The studied configuration is the one described in Section 2.1. The setup was created both experimentally (see Fig. 3) and numerically. For the in-lab experiment, a through-hole was drilled at the position $(x, y)=[250,280] \mathrm{mm}$. One acquisition was made for nine through-hole radius from 2.0 to $7.5 \mathrm{~mm}$. In Fig. 4, the image obtained using the DAS algorithm based on experimental signals for the $5.0 \mathrm{~mm}$ radius hole is shown.

A training dataset containing 350 samples was generated using CIVA with the Latin Hypercube Sampling (LHS) strategy for the variation ranges of the flaw coordinates $x$ and $y$ limited within the sensor network. Defect diameter variation range was within the interval $[2.51,7.47] \mathrm{mm}$, which is the result of a uniform random draw between $[2.5,7.5] \mathrm{mm}$. Since the signals from simulated 


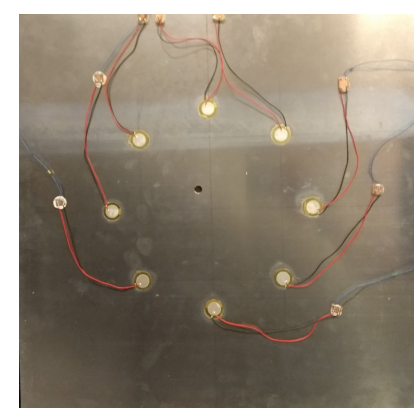

Figure 3: SHM experimental setup with in aluminium plate with eight piezoelectric sensor encircling a hole drilled within the plate.

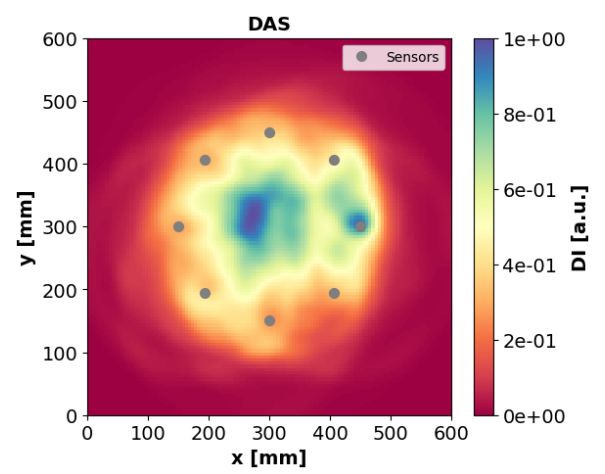

Figure 4: Experimental GW-SHM signal post processed via DAS algorithm for a 5.0 mm radius hole.

and experimental data do not share the same scale, the amplitude of the simulated signals are calibrated based on the signals from the healthy structure of the experimental data. Such a way of calibrating signals allow them to share the same scale enabling the direct comparison of the signals.

Referring to the quantities introduced in the section 4 the training set contains $N=350$ images made of $M=14400$ pixels (i.e., $\mathbf{X}_{t r n} \in \mathbb{R}^{350 \times 14440}$ ) for the regression tasks. For classification purposes the number of images are doubled since 350 images representative of a pristine plate have been added to the training set via the noise model introduced in Section 2.1. In order to fit the SVM model for classification and inversion tasks the training set is first projected into the latent feature space via PCA algorithm by choosing an explained variance (see equation (10)) equal to $98 \%$ of the training set. That is, for classification task, the number of extracted features $J=12$ and the training set size was equal to $\tilde{\mathbf{X}}_{t r n}^{S V C} \in \mathbb{R}^{700 \times 12}$ (i.e., 350 pristine and 350 damage samples). For the regression task, $J=9$ thus the training set obtained was $\tilde{\mathbf{X}}_{t r n}^{S V R} \in \mathbb{R}^{350 \times 9}$ have been considered for the classification and the regression tasks, respectively. The difference between these two values is due to the fact that, for regression tasks, the laboratory measurements were not corrupted by thermal and electronic noise as well as temperature change. Therefore, the value $\nu$ in (1) within the training set is chosen equal to zero. On the other hand, the simulated pristine signals added in the training set for classification were chosen with $\nu$ equal to 0.02 in order to create images embedding artefacts appearing as flaw-like signals. The training set $\tilde{\mathbf{X}}_{t r n}$ is then fitted to a SVC model by employing a 5-fold cross-validation stage to get the best possible $C$ and $\sigma$ parameters. In the case of regression tasks, the 5 -fold cross-validation was performed on $\varepsilon$ and $C$ SVR parameters and on kernel parameter $\sigma$ all together. Results of the cross-validation procedure is shown in Fig. 5 for what concerns the training of the SVC model based on the $f 1$-score. This 


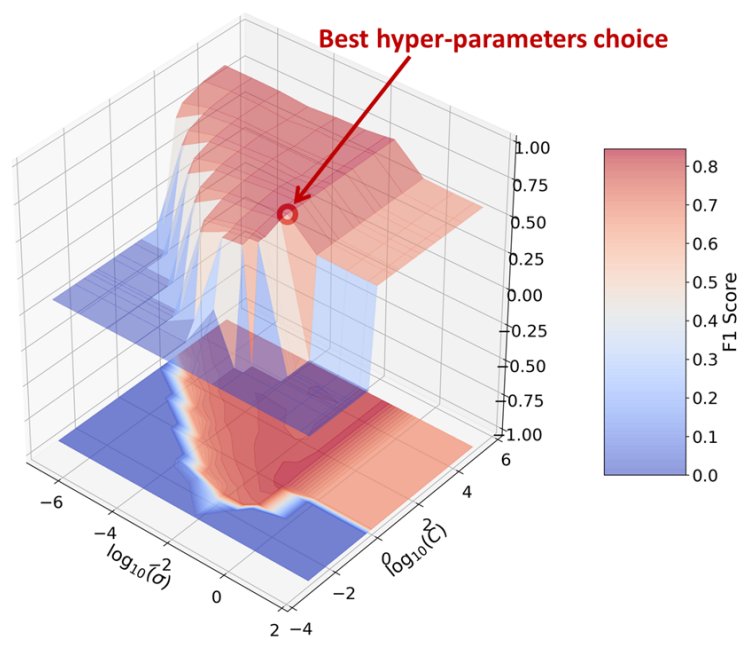

Figure 5: SVM cross-validation classification results shown in terms of f1-score values provided on a the tensor grid obtained for different values of $C$ and $\sigma$.

figure shows that only a subset of the cross-validation grid maximize the $f 1$-score and that, on the boundaries of the hyper-parameters domain, the performance of the classifiers decreases.

\subsection{Support vector machine classification results}

Based on the above training strategy, the SVM classifier is tested for two classes problem targeting flaw detection (i.e., flawless vs. flawed specimen) tasks. The results obtained by applying the aforementioned ML strategy are shown in Fig. 6. In Fig. 6a), it can be observed that the SVM classifier is able to properly classify the flawed specimens with a $96 \%$ success rate, whereas it performs at $95 \%$ for the classification of flawless specimens. That is, following the approach provided in [34], the confusion matrix in Fig. 6a) corresponds to the point on the Receiving Operating Characteristic (ROC) curve in Fig. 6b) associated to the SVC classifier with true positive and false positive values equal to 0.96 and 0.04, respectively. Still in Fig. 6b), the ROC curve is provided along with the estimated Area Under the ROC Curve (AUC) that, for the SVC algorithm, is equal to about 0.98. In the same plot, in order to compare the SVC results with respect a "standard" yet effective classifier, the classifications results obtained via Naive Bayes (NB) [35] is overlapped to the SVC ROC curve. Both these results shown that discrimination between flawless and flawed classes obtained via SVC can be judged to perform very good [34] and SVC provides better results compared to NB. In Section 8, we provide a further analysis on the classification results obtained for three different flaws categories sorted in terms of flaws sizes.

\subsection{Support vector machine regression results}

In this section, regression results in terms of inversion to retrieve flaw position and radius size based on both synthetic and experimental tests sets are presented. In addition to the training set, a test set consisted in $T=141$ samples is created under the same conditions. Furthermore the $T=8$ experiments previously described are also considered. After projecting of both the test sets onto the PCA extracted feature space by employing Equation (9), the test set size for the synthetic data and experiments are $\tilde{\mathbf{X}}_{t s t} \in \mathbb{R}^{141 \times 9}$ and $\tilde{\mathbf{X}}_{t s t} \in \mathbb{R}^{8 \times 9}$, respectively. In Fig. 7, predictions given by the SVM regressor are provided in terms of true versus predicted values. The predictions based on experimental data ("x") are overlapped to the numerical one ("•"). As one can notice, in the case of numerical data, the scattered points are aligned to the diagonal solid line 


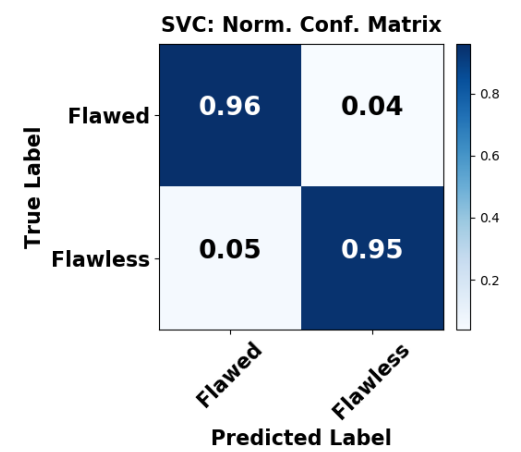

a)

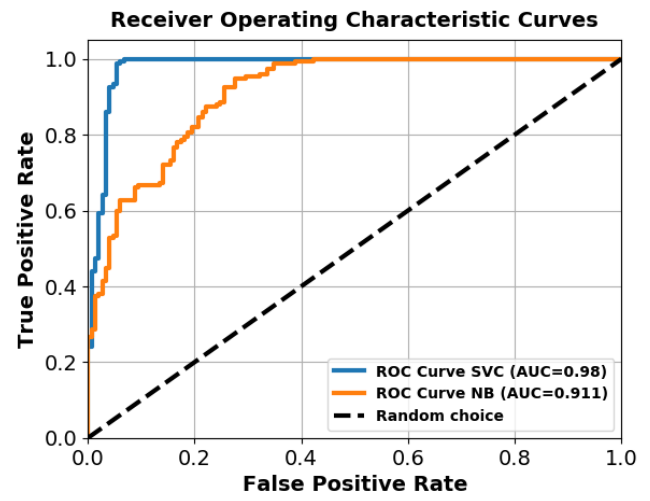

b)

Figure 6: Classification results obtained via SVM classifier are shown in terms of $a$ ) normalized confusion matrix and $b$ ) ROC curve with all flaws radius sizes considered. 


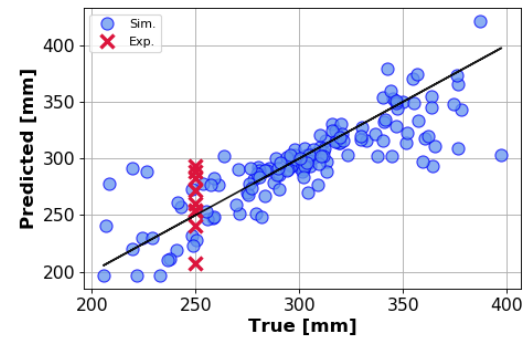

a)

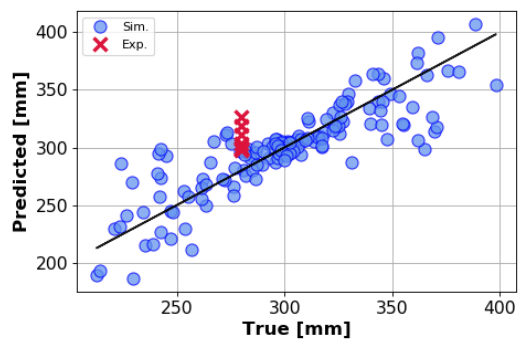

b)

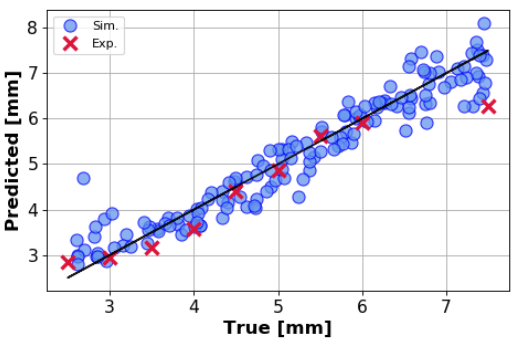

c)

Figure 7: SVM regression results obtained for flaw a) $x$-position, $b$ ) $y$-position and $c$ ) radius where " $\bullet "$ indicates prediction based on synthetic data (with $\nu$ equal to zero) whereas " $\times$ " stands for prediction based on experimental data.

that represents the perfect agreement between predictions and ground truth. On the other hand, experimental data regression is slightly more spread for flaw positions compared to the synthetic counterpart. Nevertheless, the accuracy in radius characterization is within the scattered cloud of points associated to the synthetic test set. Concerning the experimental test set, it worth mentioning that predictions having the two largest outliers are associated to points that are either on the training set boundaries (radius equal to $7.5 \mathrm{~mm}$ ) or even outside (radius equal to $2.5 \mathrm{~mm}$ ) the training set data samples. This behaviour is clear in Fig. 7 for the smallest and the largest radius.

From a quantitative point of view, the accuracy of the predictions can be quantified using the Mean Absolute Error (MAE) defined as $M A E=\sum_{i=1}^{T}\left|y_{i}-\hat{y_{i}}\right| / T$ and the Root Mean Squared Error (RMSE) metric defined as $R M S E=\sqrt{\sum_{i=1}^{T}\left(y_{i}-\hat{y}_{i}\right)^{2} / T}$. In Fig. 8, is displayed a study of robustness of the SVR model with respect to different level of arificial noise $\nu$. Such scenario may appear when the predictions data (i.e., the test set) are based on data acquired under slightly different experimental conditions compared than the training set. The results in Fig. 8, showed that increasing the value of $\nu$ increases both the MAE and RMSE. Among all parameters, flaw diameter is the one that suffer more of such a kind of noise corruption with MAE equal to $0.29 \mathrm{~mm}$ at $\nu=0.0$ to MAE equal to $2.66 \mathrm{~mm}$ at $\nu=3.0 e-2$. This behaviour is due to the fact that very low changes in the $\nu$ translate in possibly heavy changes in the GWIs. A way to mitigate such an issue, consists in considering an alternative training set containing different values of $\nu$, and in general, different values for the parameters of the noise model, which should lower the errors observed in Fig. 8.

From a computational point of view, the 156 predictions are carried out in about $5 \mathrm{msec}$ on an Intel Xeon E3 - 1505M processor with 32 GB of RAM laptop. Furthermore, as for the classification tasks, the results are computed only based on the knowledge of the PCA transformation matrix (9) and the values of the support vectors and bias in (17) matrix. Therefore, a very low computational burden is associated to the prediction phase which leads to a CPU time of about 3 msec to predict all test set samples. That is, the employed matrices are lightweight pre-computed numerical objects (i.e., about 29 megabytes) that can be easily embed and handled inside latests generation portable inspection devices or in acquisition chain enabling online automatic quasi real-time classification and regression tasks. 
MAE vs. $v$

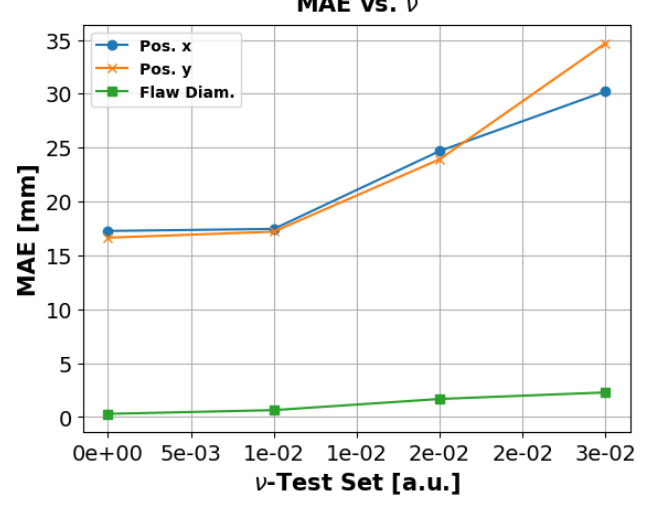

a)

RMSE vs. $v$

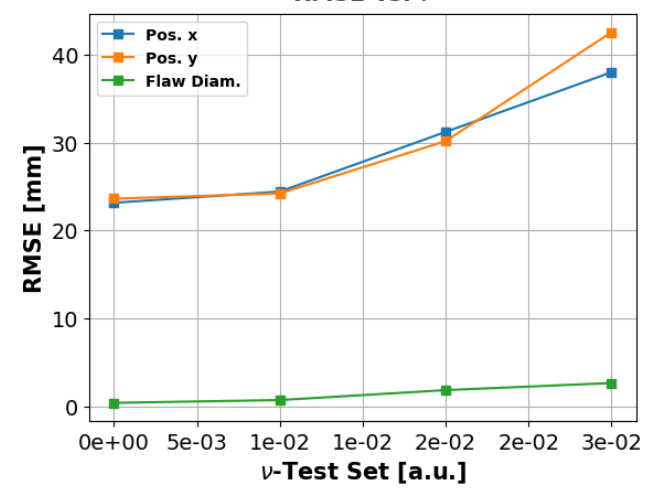

b)

Figure 8: Study of robustness of SVM regression with respect to different values of the amplitude of the noise level 1. In $a$ ) in terms of $M A E$ coefficient and in $b$ ) in terms of $R M S E$ for flaw $x$-position, $y$-position and diameter. 


\section{Conclusion and perspectives}

In this paper, a machine learning framework dedicated to flaw(s) detection, localization and characterization applied to both structural health monitoring measurements and synthetic data is presented. In particular, performance associated to damage detection have been assessed based on synthetic data corrupted with a realistic noise model aiming at reproducing a realistic texture in the GW images. Concerning the regression task, i.e., flaw localization and characterization, the proposed methodology is tested on both noisy synthetic data and experimental measurements. For both the classification and regression tasks, good results have been found in terms of accuracy and a high CPU time efficiency have been obtained such that the developed procedure can provide real time predictions.

The next research axes are mainly devoted to perform a broader experimental campaign targeting the validation of the ML strategy proposed on different kind of flaws possibly embedded in different kind of medium e.g., composite materials and structures. Due to the more complex ultrasounds propagation phenomena in such a kind of media, the proposed strategy may require modifications in order to extract the most meaningful information from the post-processed GW signals. In this framework, non linear feature extraction methods [36], vector valued extension of SVM and deep learning regression [37] will be also considered.

\section{Appendix 1: complementary ROC analysis}

In this Appendix, a complementary classification result is presented by analysing the performance of SVC and NB classifiers for different flaws size. Toward this end, the test set is sorted by considering three different flaw categories based on the flaw radius size. That is, the flaws with radius between $2.51 \mathrm{~mm}$ and $4.17 \mathrm{~mm}, 4.17 \mathrm{~mm}$ and $5.83 \mathrm{~mm}$ and $5.83 \mathrm{~mm}$ and $7.47 \mathrm{~mm}$ have been considered. Through the scatter plot matrix in Fig. 9, the test set GWIs projected into the first four PCA (latent space) dimensions for the three considered flaws categories are shown. One can notice that the overlap between classes is higher for flaws smaller than $3.8 \mathrm{~mm}$ whereas it slightly decreases for larger flaws. The absence of two distinct clusters in the PCA latent space underlines the fact that the noise model adopted in this paper is able to reproduce pristine DAS GWIs that "looks like" damaged one. Therefore, non-trivial separation hyperplane and classification rules must be established for the three considered flaw categories.

In Fig. 10, the results obtained via SVC and NB algorithms are shown. Overall for both classification methods, one can notice that larger defects (i.e. radius larger than about $4.17 \mathrm{~mm}$ ) are detected with high accuracy. Furthermore, SVC provide superior results than NB classifier regardless of the flaw size considered. The higher SVC's performance are obtained thanks to the non-linear model capabilities thanks to the use of the kernel trick (see Section 5.2).

\section{Appendix 2: classification and regression with temperature compensation}

The purpose of this appendix is to study the impact on classification and regression performance once the Baseline Signal Stretch (BSS) algorithm is applied to raw GW signals. As a reminder, the baseline signal stretch modifies the signals in the time domain by stretching or compressing the time axis. This is done in order to compensate the variation of the group velocity of the GW due to a temperature change. For more information on BSS, the reader is invited to refer to [9].

In Fig. 11 the results obtained by SVC and NB trained on DAS GWIs after applying the BSS correction on the raw signals are provided. The results obtained in Fig. 11 are very close to the one provided in 6. That is, performing BSS correction on GW raw signals lead to almost no 

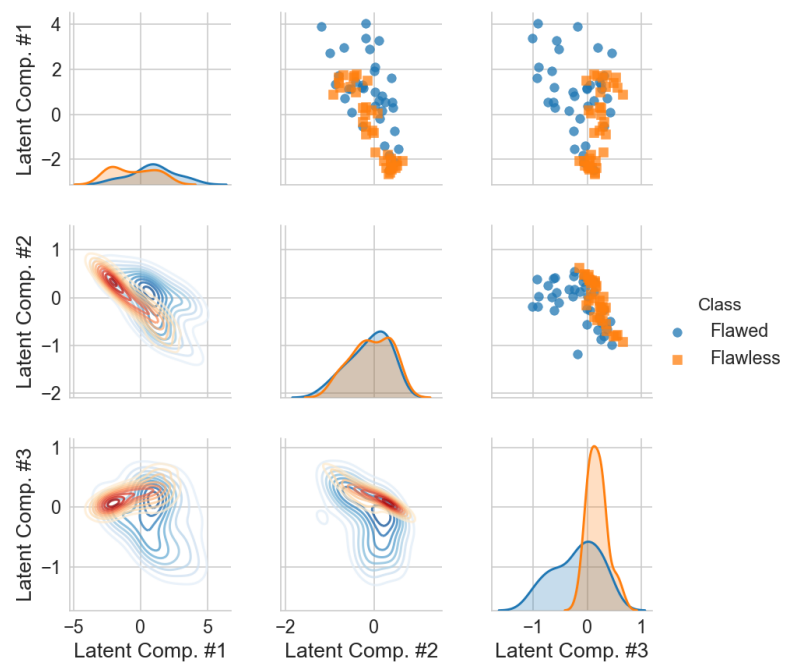

a)
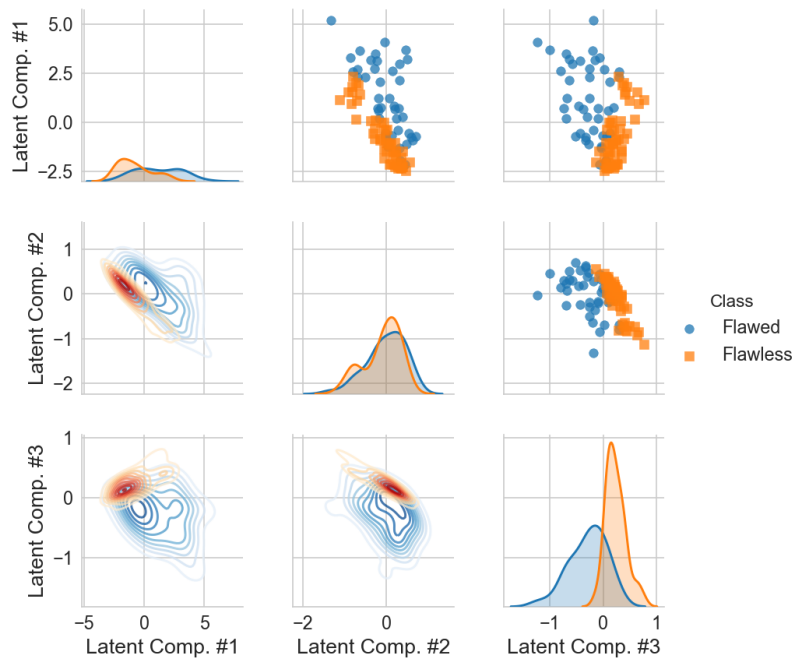

b)
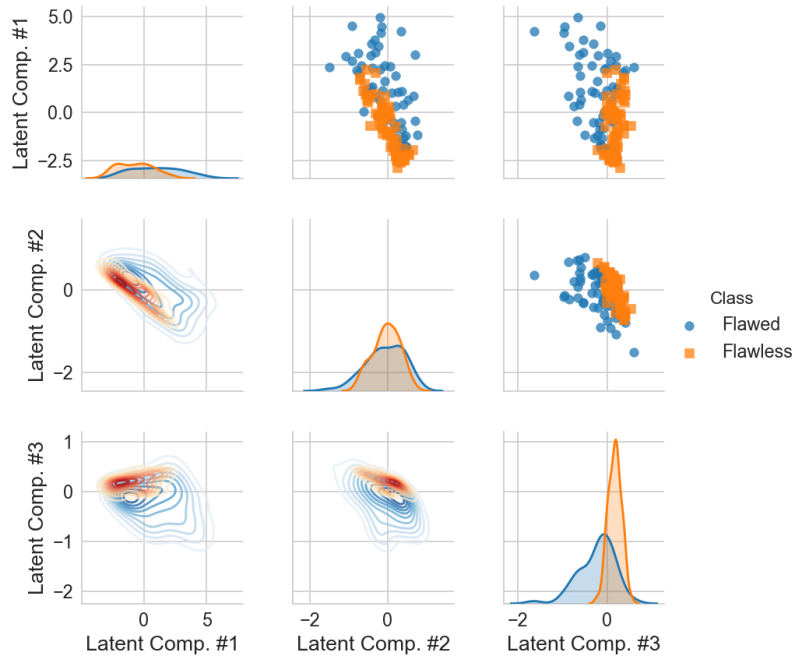

c)

Figure 9: ROC curves obtained via SVC and Naive Bayes classifiers are shown for flaw radius in $a$ ) between $2.51 \mathrm{~mm}$ and $4.17 \mathrm{~mm}$, in $b$ ) between $4.17 \mathrm{~mm}$ and $5.83 \mathrm{~mm}$ and in $c$ ) between $5.83 \mathrm{~mm}$ and $7.47 \mathrm{~mm}$. 


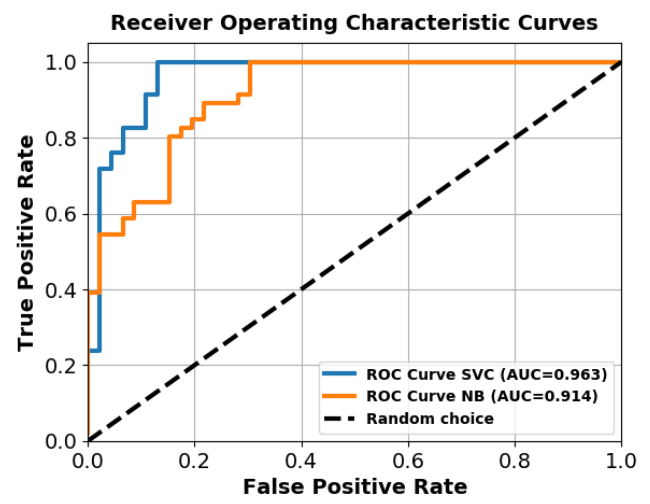

a)

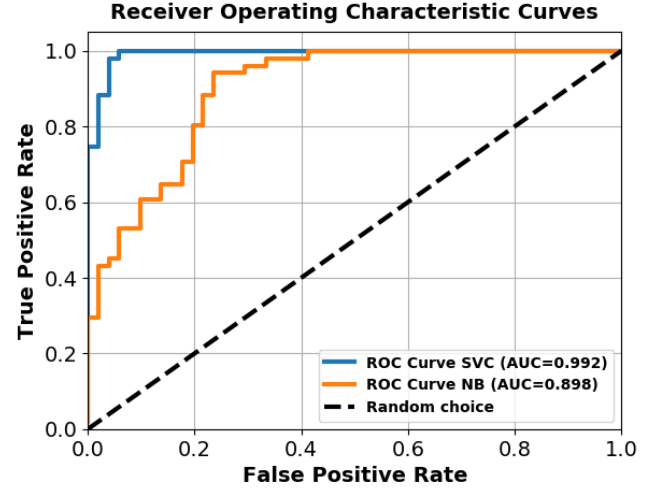

b)

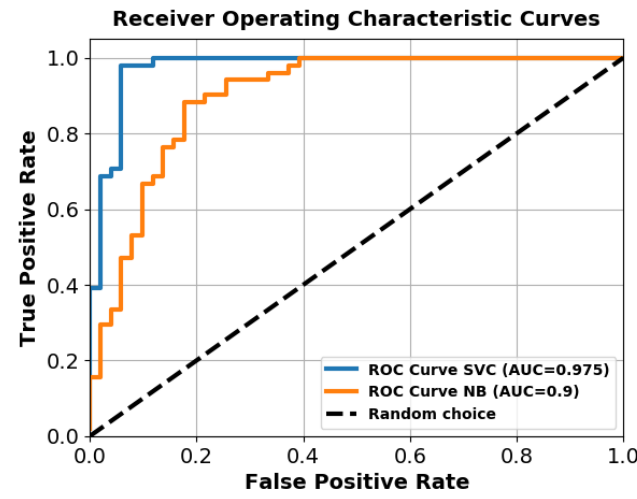

c)

Figure 10: ROC curves obtained via SVM and Naive Bayes classifiers are shown for flaw radius in $a$ ) between $2.51 \mathrm{~mm}$ and $4.17 \mathrm{~mm}$, in $b$ ) between $4.17 \mathrm{~mm}$ and $5.83 \mathrm{~mm}$ and in $c$ ) between $5.83 \mathrm{~mm}$ and $7.47 \mathrm{~mm}$. 


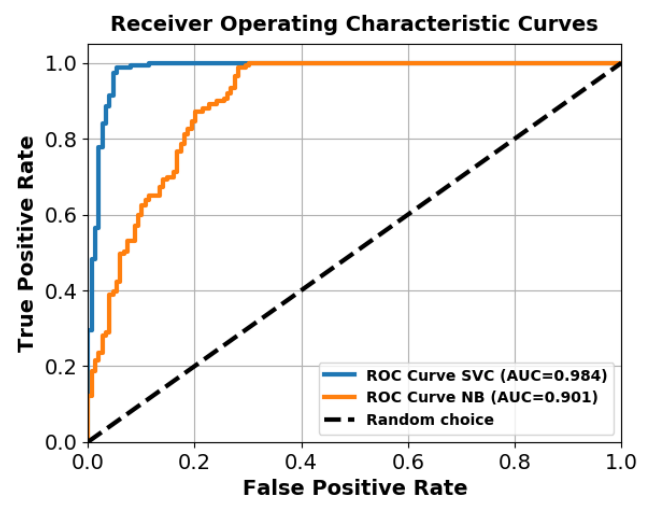

b)

Figure 11: ROC curve results (with all flaws radius sizes considered) obtained with NB and SVC algorithms based on raw GW signals corrected with BSS.

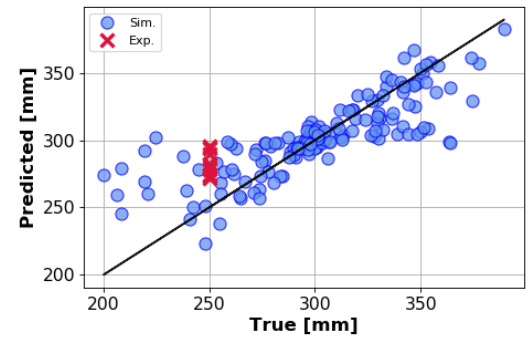

a)

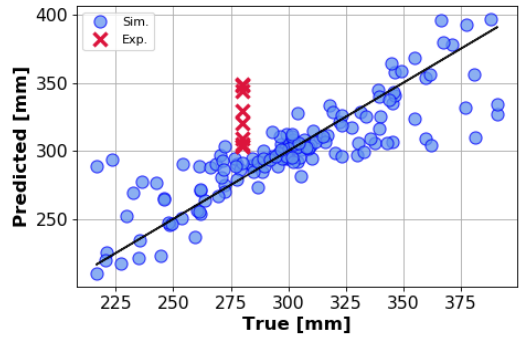

b)

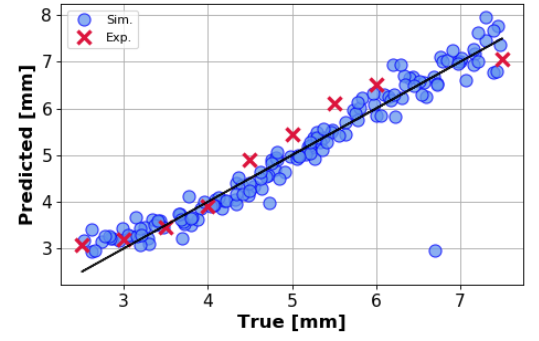

c)

Figure 12: SVM regression results based on BSS corrected signals before performing imaging are obtained for flaw a) $x$-position, $b$ ) $y$-position and c) radius where "•" indicates prediction based on synthetic data (with $\nu$ equal to zero) whereas " $\times$ " stands for prediction based on experimental data.

improvements on the classification performance. This result is not a surprise, indeed BSS is used to obtain images with sharp flaw indications for easier human interpretation. However, the SVM can achieve successful data interpretation even without BSS because it conducts its analysis on more abstract features that would be meaningless for a human. In other words, with and without BSS, the information about the defect is contained in the GWI and the SVM can analyse it in both cases, however a human would need BSS to analyse robustly the images.

In Fig. 12, the regression results based on the use of SVM trained on GWIs generated via BSS corrected GW raw signals are presented. Compared to Fig. 7, one can notice that the use of BSS provides almost no improvements in the inversion performance. The MAE is equal to $16.52 \mathrm{~mm}$, $15.31 \mathrm{~mm}$ and $0.24 \mathrm{~mm}$ for the hole position along $\mathrm{x}, \mathrm{y}$ and the hole radius size, respectively. In terms of RMSE, the result is $23.56 \mathrm{~mm}, 22.73 \mathrm{~mm}$ and $0.41 \mathrm{~mm}$ for the hole position along $\mathrm{x}, \mathrm{y}$ and the hole radius size, respectively.

The results presented in this section, underline that the joint use of feature extraction and kernel-based SVM methods applied on GWIs are able to handle, up to some extent, the effects of temperature variations and electronic noise corruptions on GW raw signals bypassing the use of signal correction before generating GWIs. 


\section{References}

[1] M. Mitra, S. Gopalakrishnan, Guided wave based structural health monitoring: A review, Smart Materials and Structures 25 (5) (2016) 053001.

[2] M. Gresil, V. Giurgiutiu, Guided wave propagation in composite laminates using piezoelectric wafer active sensors 117 (1196) (2013) 971-995.

[3] X. Zhao, H. Gao, G. Zhang, B. Ayhan, F. Yan, C. Kwan, J. L. Rose, Active health monitoring of an aircraft wing with embedded piezoelectric sensor/actuator network: I. defect detection, localization and growth monitoring 16 (4) (2007) 1208.

[4] J. E. Michaels, Detection, localization and characterization of damage in plates with an in situ array of spatially distributed ultrasonic sensors 17 (3) (2008) 035035.

[5] J. S. Hall, P. Fromme, J. E. Michaels, Guided wave damage characterization via minimum variance imaging with a distributed array of ultrasonic sensors 33 (3) (2014) 299-308.

[6] N. Quaegebeur, P. Ostiguy, P. Masson, Correlation-based imaging technique for fatigue monitoring of riveted lap-joint structure 23 (5) (2014) 055007.

[7] A. Kulakovskyi, O. Mesnil, A. Lhémery, B. Chapuis, O. d'Almeida, Defect imaging in layered composite plates and honeycomb sandwich structures using sparse piezoelectric transducers network, in: Journal of Physics: Conference Series, Vol. 1184, IOP Publishing, 2019, p. 012001.

[8] A. Recoquillay, T. Druet, S. Nehr, M. Horpin, O. Mesnil, B. Chapuis, G. Laffont, O. D'Almeida, Guided wave imaging of composite plates using passive acquisitions by fiber bragg gratings, The Journal of the Acoustical Society of America 147 (5) (2020) 3565-3574.

[9] G. Liu, Y. Xiao, H. Zhang, G. Ren, Baseline signal reconstruction for temperature compensation in lamb wave-based damage detection 16 (8) (2016) 1273.

[10] A. J. Croxford, J. Moll, P. D. Wilcox, J. E. Michaels, Efficient temperature compensation strategies for guided wave structural health monitoring 50 (4) (2010) 517-528.

[11] A. Douglass, J. B. Harley, Dynamic time warping for temperature compensation in structural health monitoring 1806 (1) (2017) 020017.

[12] P. Huthwaite, F. Simonetti, High-resolution guided wave tomography 50 (5) (2013) 979-993.

[13] E. M. Tom Druet, Bastien Chapuis, Passive guided wave tomography for corrosion detection, 2016.

[14] R. Levine, Ultrasonic guided wave imaging via sparse reconstruction (2014).

[15] O. Mesnil, M. Ruzzene, Sparse wavefield reconstruction and source detection using compressed sensing 67 (2016) 94-104.

[16] J. Moriot, N. Quaegebeur, A. Le Duff, P. Masson, A model-based approach for statistical assessment of detection and localization performance of guided wave-based imaging techniques (2017) 1475921717744679. 
[17] L. Bai, A. Velichko, B. W. Drinkwater, Characterization of defects using ultrasonic arrays: a dynamic classifier approach 62 (12) (2015) 2146-2160. doi:10.1109/TUFFC.2015.007334.

[18] N. Munir, H.-J. Kim, J. Park, S.-J. Song, S.-S. Kang, Convolutional neural network for ultrasonic weldment flaw classification in noisy conditions, Ultrasonics 94 (2019) 74-81.

[19] M. Salucci, N. Anselmi, G. Oliveri, P. Calmon, R. Miorelli, C. Reboud, A. Massa, Realtime NDT-NDE through an innovative adaptive partial least squares SVR inversion approach 54 (11) (2016) 6818-6832. doi:10.1109/TGRS.2016.2591439.

[20] S. Ahmed, M. Salucci, R. Miorelli, N. Anselmi, G. Oliveri, P. Calmon, C. Reboud, A. Massa, Real time groove characterization combining partial least squares and SVR strategies: application to eddy current testing 904 (2017-10) 012017. doi:10.1088/1742-6596/904/1/012017.

[21] C. Willberg, S. Duczek, J. M. Vivar-Perez, Z. A. B. Ahmad, Simulation methods for guided wave-based structural health monitoring: A review 67 (1) (2015) 010803.

[22] O. Mesnil, A. Imperiale, E. Demaldent, V. Baronian, B. Chapuis, Simulation tools for guided wave based structural health monitoring, in: AIP Conference Proceedings, Vol. 1949, AIP Publishing, 2018, p. 050001.

[23] O. Mesnil, A. Imperiale, E. Demaldent, B. Chapuis, Validation of spectral finite element simulation tools dedicated to guided wave based structure health monitoring, in: AIP Conference Proceedings, AIP Publishing, 2019.

[24] A. Imperiale, E. Demaldent, A macro-element strategy based upon spectral finite elements and mortar elements for transient wave propagation modeling. application to ultrasonic testing of laminate composite materials, International Journal for Numerical Methods in Engineering 119 (10) (2019) 964-990.

[25] G. Cohen, Higher-Order Numerical Methods for Transient Wave Equations, Springer: Scientific Computation, 2002.

[26] E. F. Crawley, J. De Luis, Use of piezoelectric actuators as elements of intelligent structures, AIAA journal 25 (10) (1987) 1373-1385.

[27] F. Le Bourdais, O. Mesnil, O. d'Almeida, Machine-learning based temperature compensation for guided wave imaging in structural health monitoring (2019).

[28] V. Giurgiutiu, Tuned lamb wave excitation and detection with piezoelectric wafer active sensors for structural health monitoring, Journal of intelligent material systems and structures 16 (4) (2005) 291-305.

[29] E. Kausel, Wave propagation in anisotropic layered media 23 (8) (1986) 1567-1578.

[30] N. Cristianini, J. Shawe-Taylor, An introduction to support vector machines and other kernelbased learning methods, Cambridge university press, 2000.

[31] I. T. Jolliffe, Principal component analysis, Springer series in statistics, Springer-Verlang, 1986. 
[32] J. S.-T. N. Cristianini, Kernel Methods for Pattern Analysis, Cambridge University Press, 2004.

[33] V. Kecman, Support vector machines - an introduction, in: L. Wang (Ed.), Support Vector Machines: Theory and Applications, Studies in Fuzziness and Soft Computing, Springer Berlin Heidelberg, 2005, pp. 1-47.

[34] T. Fawcett, An introduction to ROC analysis 27 (8) (2006) 861-874.

[35] H. Zhang, The optimality of naive bayes, in: V. Barr, Z. Markov (Eds.), Proceedings of the Seventeenth International Florida Artificial Intelligence Research Society Conference (FLAIRS 2004), AAAI Press, 2004.

[36] M. Salucci, N. Anselmi, G. Oliveri, P. Rocca, S. Ahmed, P. Calmon, R. Miorelli, C. Reboud, A. Massa, A nonlinear kernel-based adaptive learning-by-examples method for robust NDT/NDE of conductive tubes 33 (6) (2019) 669-696. doi:10.1080/09205071.2019.1572546.

[37] P. Zhu, Y. Cheng, P. Banerjee, A. Tamburrino, Y. Deng, A novel machine learning model for eddy current testing with uncertainty 101 (2019) 104-112. doi:10.1016/j.ndteint.2018.09.010. 